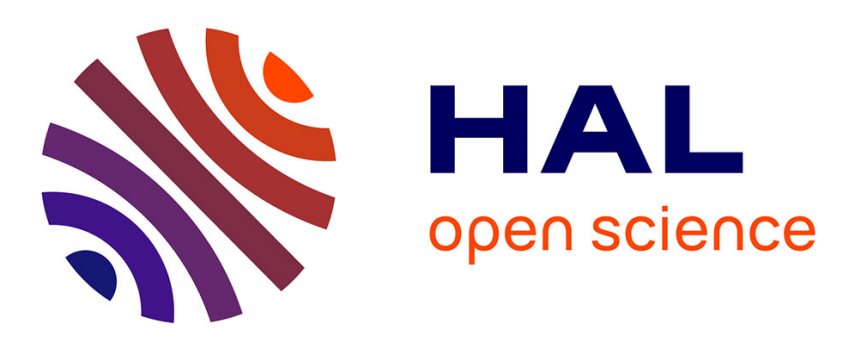

\title{
Forums de discussion : médiations et écriture de recherche
}

\author{
Georges Ferone, Richard-Principalli Patricia
}

\section{To cite this version:}

Georges Ferone, Richard-Principalli Patricia. Forums de discussion : médiations et écriture de recherche. Recherche et formation, 2016, 81, pp.37-50. 10.4000/rechercheformation.2615 . hal02073399

\section{HAL Id: hal-02073399 \\ https://hal.umontpellier.fr/hal-02073399}

Submitted on 19 Mar 2019

HAL is a multi-disciplinary open access archive for the deposit and dissemination of scientific research documents, whether they are published or not. The documents may come from teaching and research institutions in France or abroad, or from public or private research centers.
L'archive ouverte pluridisciplinaire HAL, est destinée au dépôt et à la diffusion de documents scientifiques de niveau recherche, publiés ou non, émanant des établissements d'enseignement et de recherche français ou étrangers, des laboratoires publics ou privés. 


\section{Recherche et formation}

$81 \mid 2016$

Varia

\section{Forums de discussion : médiations et écriture de recherche}

Discussion forums: mediation and research writing

\section{Georges Ferone et Patricia Richard-Principalli}

revues.org

Édition électronique

URL : http://

rechercheformation.revues.org/2615

DOI : 10.4000/rechercheformation.2615

ISSN : 1968-3936

\author{
Éditeur \\ ENS Éditions \\ Édition imprimée \\ Date de publication : 30 avril 2016 \\ Pagination : $37-50$ \\ ISSN : 0988-1824
}

Distribution électronique Cairn

CAIRN IN FO

CHERCHER, REPÉRER, AVANCER,

Référence électronique

Georges Ferone et Patricia Richard-Principalli, « Forums de discussion : médiations et écriture de recherche », Recherche et formation [En ligne], 81 | 2016, mis en ligne le 30 avril 2018, consulté le 27 octobre 2017. URL : http://rechercheformation.revues.org/2615 ; DOI : 10.4000/

rechercheformation. 2615 


\section{Forums de discussion : médiations et écriture de recherche}

\section{$>$ Georges Ferone}

Université Paris-Est Créteil Val-de-Marne (UPEC), Centre interuniversitaire de recherche, culture, éducation, formation et travail (CIRCEFT, EA 4384), équipe Éducation et scolarisation (ESCOL); université Paris 8 Vincennes-Saint-Denis

\section{$>$ Patricia Richard-Principalli}

Université Paris-Est Créteil Val-de-Marne (UPEC), Centre interuniversitaire de recherche, culture, éducation, formation et travail (CIRCEFT, EA 4384), équipe Éducation et scolarisation (ESCOL); université Paris 8 Vincennes-Saint-Denis

RÉSUMÉ : En formation d'enseignants, des formateurs utilisent des forums pour le suivi des mémoires de recherche. L'analyse des échanges de ces forums montre l'importance et la complexité de cet accompagnement. Elle met en évidence des apports différenciés du forum selon les étudiants et montre les effets relatifs d'une médiation uniquement centrée sur les contenus. Elle souligne l'importance de la médiation réflexive dans le processus d'accompagnement pour surmonter les nombreuses tensions à l'œuvre dans l'écriture de recherche.

MOTS-CLÉS : enseignement à distance, forum, écriture, formation des enseignants

\section{Introduction}

Depuis la mastérisation de la formation survenue en 2010, les étudiants futurs enseignants, en particulier ceux du premier degré de l'académie de Créteil, doivent produire un mémoire de recherche. Du fait de ses caractéristiques ${ }^{1}$, ce genre d'écrit est difficile à produire par les étudiants (Reuter, 2004), ce qui soulève la question de l'accompagnement de la part des formateurs. Celui-ci se déroule de plus en plus souvent à distance ${ }^{2}$, notamment par le biais de forums de discussion qui permettent de développer des échanges collectifs.

L'objet de cette étude est de mieux comprendre les effets des échanges via les forums de discussion et le rôle de la médiation du formateur dans la production des

1 L'objectif de ce mémoire et les compétences qui vont de pair sont ainsi résumés par Delarue-Breton et Crinon (2014, p. 1) : "Rédiger ce mémoire a pour objectif d'amener les étudiants à s'approprier des connaissances et des cadres théoriques leur permettant d'analyser les corpus constitués sur des terrains scolaires et d'acquérir ainsi des compétences à mieux comprendre les situations d'apprentissage, d'enseignement et d'éducation auxquelles ils se trouvent professionnellement confrontés."

2 Un des effets de la mastérisation de la formation des maîtres a été de développer la formation à distance dans les ESPE, notamment dans celles de Créteil et de Versailles. 
écrits de recherche des étudiants. De nombreux travaux montrent les effets positifs de la médiation relationnelle sur la participation à un forum (Bruillard, 2010; Quintin, 2008) mais peu s'intéressent aux effets différenciés de tels échanges sur les apprentissages des étudiants. Or, à défaut d'une réelle médiation de l'enseignant, il apparaît que seuls les étudiants les plus autodirigés profitent des dispositifs d'accompagnement tels que les forums de discussion.

Dans un premier temps, les champs théoriques dans lesquels s'inscrit cette recherche sont présentés. La spécificité de l'écrit de recherche en formation d'enseignants et les obstacles rencontrés par les étudiants sont ensuite définis. Puis, est exposée une étude de cas menée dans le contexte de la formation à distance de l'ESPE de l'Académie de Créteil, basée sur l'analyse de forums de discussion.

\section{Cadre théorique}

Cette recherche s'inscrit dans quatre champs théoriques.

\subsection{Le champ des littératies universitaires et l'écriture en formation}

Les études de référence en ce domaine (Barré-De Miniac, Brissaud et Rispail, 2004; Crinon et Guigue, 2006; Delarue-Breton et Crinon, 2015; Lafont-Terranova et Niwese, 2012; Reuter, 2012) considèrent l'écriture comme une pratique sociale et mettent en évidence l'importance de l'activité de réception des scripteurs, les contraintes et les obstacles propres à l'écriture de recherche. L'activité d'écriture dans cette conception n'est plus considérée comme un ensemble de codes et de règles à maîtriser mais comme la prise en compte de "leurs conditions de possibilité, de leurs usages et de leurs effets par et pour des sujets donnés dans des contextes sociaux déterminés" (Reuter, 2012, p.162).

\subsection{Le champ de la communication médiée par ordinateur (CMO) et des apprentissages collaboratifs assistés par ordinateur (ACAO)}

Des travaux soulignent les apports de celui-ci pour la formation (Bruillard, 2010; Ferone, 2011), ils mettent notamment en évidence l'intérêt du forum de discussion pour soutenir les interactions et les apprentissages. Le forum ralentit le temps (par rapport aux interactions orales instantanées) et facilite la prise de distance. Public et interactif, il favorise la structuration des échanges et l'exposition des points de vue (Mangenot, 2002). Le forum a ainsi une fonction de mobilisation d'idées, il permet la constitution d'un répertoire de possibles, puis un tri progressif parmi les idées avancées, il facilite la décentration et permet une appropriation progressive des contenus. D'autres travaux soulignent la nécessité d'un accompagnement et d'un tutorat à distance pour favoriser les apprentissages (Depover, De Lièvre, Peraya, Quintin et Jaillet, 2011). Quatre types de médiations instrumentales sont ainsi distinguées : la médiation épistémique orientée vers la connaissance de l'objet; la médiation praxéologique orientée vers l'action; la médiation réflexive orientée vers le sujet lui-même et la médiation relationnelle qui se réalise entre les sujets (Rabardel et Samurçay, 2006). 


\subsection{Le champ de l'autodirection}

Les travaux de Jézégou (2008) et de Cosnefroy (2010) soulignent la nécessité de prendre en considération les caractéristiques de l'apprenant et de l'environnement d'apprentissage pour statuer sur l'efficacité potentielle de tout dispositif technologique. Ils mettent en évidence des configurations plus ou moins favorables aux apprentissages en fonction du niveau d'autonomie de l'apprenant et du contrôle pédagogique plus ou moins fort exercé par le dispositif pédagogique. Un contrôle pédagogique faible convient mieux à un apprenant autodirigé; à l'inverse, un contrôle pédagogique fort est plus favorable quand l'apprenant est peu autodirigé3 (Jézégou, 2008).

\subsection{Le champ des inégalités sociales de réussite scolaire et la manière dont elles se construisent à l'école et à l'université}

Nos observations nous invitent à penser que le concept de coconstruction des inégalités (Bautier et Goigoux, 2004; Bautier et Rayou, 2009, Rochex et Crinon, 2011) est transférable du contexte scolaire au contexte universitaire. Deux facteurs se conjuguent pour expliquer les inégalités d'apprentissage à l'université : d'une part, des facteurs liés au mode de socialisation des étudiants qui prédisposent plus ou moins favorablement que d'autres aux apprentissages littératiés et à l'affiliation au monde universitaire, d'autre part, des facteurs qui se jouent au sein même de l'université et qui concernent les pratiques enseignantes et les dispositifs d'apprentissage proposés, qui peuvent réduire ou au contraire aggraver les inégalités liées au mode de socialisation familiale (Coulon et Paivandi, 2008). Coulon et Paivandi notent que la difficulté des étudiants d'origine populaire à l'université s'explique en partie par le type, la fréquence et la qualité des relations avec les enseignants.

Ces différents travaux confirment que les échanges dans des forums de discussion peuvent favoriser la production d'écrits de recherche, considérée comme une pratique sociale, mais ils soulignent aussi d'éventuels obstacles pour les étudiants les plus fragiles en fonction de l'ouverture plus ou moins grande du dispositif de formation (présence ou cadrage plus ou moins fort de la part du formateur). Pour mieux comprendre ces phénomènes, nous présentons d'abord la spécificité de l'écrit de recherche en formation des maîtres, puis une étude de cas.

3 L'autodirection s'appuie sur un double ressort : la motivation et l'autorégulation. La motivation se construit à travers la perception que l'apprenant a de lui-même au regard de son projet d'apprentissage, l'autorégulation est relative aux stratégies mises en place par l'apprenant pour surveiller, contrôler et évaluer ses apprentissages. 


\section{3. Écrire un mémoire de master Métiers de l'enseignement, de l'éducation et de la formation (MEEF)}

\subsection{Objectifs et obstacles}

Delarue-Breton (2014) définit le mémoire de master MEEF, tel qu'il est pratiqué dans l'académie de Créteil, comme un nouveau genre universitaire puisque ses finalités visent à la fois celles d'un master "recherche" et celles d'un master "professionnel ". Il relève en effet "à la fois du scientifique (pour ce qui est du genre) et du professionnel (pour ce qui est de son objet : situation, contexte, phénomène étudié)" (p. 50).

L'écriture de recherche pose de nombreuses difficultés aux étudiants en formation. Reuter (2004) identifie sept types de dysfonctionnements récurrents : des imprécisions, des modalités énonciatives inadaptées, l'absence de fonctionnalisation de certains passages, des tendances à la juxtaposition, des déséquilibres, des dysfonctionnements dans l'insertion du discours d'autrui, des problèmes de lisibilité. Pour l'écriture en formation professionnelle des enseignants, deux types de difficultés requièrent, de notre point de vue, une attention particulière, la difficulté à produire le genre d'écrit attendu ainsi que la difficulté à s'appuyer et à prendre en compte les sources et le discours d'autrui. Reuter suggère de porter attention à ces "manques" et d'envisager un enseignement spécifique à l'écriture de recherche.

\subsection{Rôle du forum dans la secondarisation attendue}

Une étude précédente ${ }^{4}$, portant sur des écrits d'étudiants (forums et mémoires) a permis, en s'appuyant sur certains des critères utilisés par Reuter (2004) et LafontTerranova et Niwese (2012), de mesurer la capacité des étudiants à "secondariser" : dialogue ou absence de dialogue avec les sources, présence ou absence de référents théoriques, conceptualisation ou subjectivité, clarté ou confusion des notions. Deux profils d'étudiants ont été identifiés, ceux qui ont des difficultés à adopter la posture réflexive attendue (absence de secondarisation) et ceux dont le processus de réflexion qui est visée par le mémoire de recherche est déjà bien engagé. L'observation $a$ posteriori de la médiation des formateurs a montré qu'ils intervenaient peu, qu'ils ne proposaient aucun étayage et s'abstenaient de toute forme d'institutionnalisation des savoirs étudiés. Ces observations permettent d'avancer que le concept de coconstruction des inégalités d'apprentissage s'applique au contexte universitaire aussi bien que scolaire.

Bautier et Goigoux (2004) ont mis en évidence le caractère implicite des attentes scolaires, ce qui génère de nombreux malentendus et différencie fortement les élèves,

4 Voir Richard-Principalli, P., Ferone, G. et Delarue-Breton, C. (2017). Writing in Discussion Forums: Between Primary Genre and Secondary Genre. Research on Writing: Multiple perspectives. Edited by Sylvie Plane, Charles Bazerman, Fabienne Rondelli, Christiane Donahue, Arthur N. Applebee, Catherine Boré, Paula Carlino, Martine Marquilló Larruy, Paul Rogers \& David Russell. Fort Collins, Colorado : WAC Clarinhouse. En ligne : <https://wac. colostate.edu/books/wrab2014> consulté en avril 2017. 
ceux capables malgré cette opacité d'identifier les enjeux cognitifs et ceux qui se limitent à l'effectuation de la tâche. On peut considérer que cette coconstruction est également à l'œuvre dans les forums étudiés. Si certains étudiants comprennent les enjeux des échanges via le forum, à l'inverse, les étudiants les plus fragiles se contentent pour la plupart de répondre à l'exercice sans en percevoir les finalités qui ne sont jamais explicitées. L'absence de formalisation des savoirs empêche en outre un transfert que les enseignants considèrent sans doute comme allant de soi. La coconstruction des inégalités est bien à l'œuvre. D'un côté, les étudiants déjà entrés dans le processus de secondarisation, ceux qui ont compris les enjeux de la situation de formation, progressent grâce au forum qui leur permet de s'exercer à l'écriture de recherche, de tester leurs analyses et d'intégrer le point de vue des autres et les ressources données dans les cours; de l'autre côté, les étudiants les plus fragiles ne profitent pas de cette modalité de formation, le forum ne faisant que refléter leur incapacité à secondariser, ce que leur écrit de recherche confirme.

Ces observations soulignent l'importance et la complexité de l'accompagnement effectué par l'enseignant dans les forums de discussion visant des objectifs de formation. Pour mieux comprendre les effets de la médiation de l'enseignant, nous avons conçu et analysé un nouveau dispositif d'accompagnement susceptible de renforcer la présence du formateur.

\section{4. Étude de cas}

\subsection{Contexte}

Dans le cadre d'une UE de recherche ${ }^{5}$ des étudiants en M1 (modalité formation à distance du Master MEEF de l'académie de (réteil) devaient, dans un premier temps, identifier les caractéristiques de ce support, puis dans un second temps, analyser de manière approfondie une page écran du support en prenant appui sur les contenus d'un cours spécifique qui proposait, de manière structurée, une analyse linguistique de pages de manuels scolaires, et anticiper d'éventuels obstacles à la compréhension que pourraient rencontrer les élèves. Ces analyses devaient être publiées dans un forum puis réutilisées pour leur note de recherche. Un formateur (extérieur à l'équipe de recherche) devait animer ce forum et produire, en guise d'institutionnalisation des connaissances, une synthèse des analyses et les commenter.

L'étude porte sur l'analyse des productions écrites des étudiants (les messages postés dans les deux forums et les notes de recherche).

5 L'objet de l'UE portait sur la "littératie numérique scolaire" (Ferone, Richard-Principalli et Crinon, 2016). Les étudiants ont été amenés à observer les caractéristiques des supports numériques, leur prise en compte par les enseignants et les effets sur les apprentissages des élèves. Les étudiants devaient traiter dans leur mémoire une question de recherche relative à ce sujet. 


\subsection{Données et méthodologie}

Nous avons pu collecter l'ensemble des données de douze étudiants (sur 21 participants), soit leurs deux analyses publiées dans les forums et leur note de recherche. Le corpus est donc constitué de vingt-quatre messages (12 pour chaque forum) et de douze notes de recherche.

Compte tenu des caractéristiques du genre visé, défini plus haut, qui suppose "un discours argumentatif à visée épistémique, dans lequel le sujet scripteur se positionne par rapport à d'autres travaux et s'affirme par le biais du travail langagier sur l'objet qu'il contribue à construire" (Lafont-Terranova et Niwese, 2012, p.5), nous avons analysé les écrits en fonction de l'usage que les étudiants font des apports théoriques. Nous distinguons :

- les apports de deux cours sur les littératies numériques (désignés "cours A»); ces cours, proposés bien en amont du travail demandé, font un état des lieux de la recherche concernant la définition et les caractéristiques de la littératie numérique;

- les apports d'un cours sur les caractéristiques linguistiques des supports "composites" (désigné "cours B"). Ce cours a été conçu précisément pour enrichir l'analyse des étudiants concernant l'analyse du support numérique, en proposant un exemple d'analyse d'un manuel papier. Le travail attendu est un travail de transfert, tant au niveau des connaissances apportées qu'au niveau de la méthodologie proposée;

- les apports de chercheurs, qu'ils figurent dans ces cours (concepts développés associés à des travaux particuliers) ou qu'ils soient les auteurs d'articles complémentaires mis à disposition des étudiants.

Nous avons ainsi observé trois productions pour les douze étudiants retenus : le forum 1, où sont identifiés les apports de chercheurs et les apports du cours A; le forum 2, où sont identifiés les apports du cours B, et d'éventuels autres apports de chercheurs; l'écrit de recherche, où sont identifiés les trois types d'apports (cours A, cours B, chercheurs), mais aussi, dans la perspective de l'étude de l'effet du forum médié, l'apport spécifique de l'enseignante en conclusion du forum 2. Nous appuyant sur les travaux de Lafont-Terranova et Niwese (2012), nous avons relevé pour chaque écrit et chaque étudiant : la présence ou l'absence des trois sources; leur tissage ou leur usage ponctuel; la pertinence de leur usage pour construire une réflexion.

Notre objectif est ainsi d'identifier dans quelle mesure la médiation de l'enseignant permet aux étudiants de prendre en compte les apports théoriques dans leurs écrits de recherche.

\subsection{Résultats et discussion}

Les choix énonciatifs effectués par les étudiants relèvent d'une écriture de type académique, celle attendue en formation : écrits structurés, syntaxe et orthographe appropriées, prise en compte du contexte de communication, visée argumentative. 
La modalité de l'exercice conditionne les échanges, il s'agit surtout d'exposer sa propre analyse du support plutôt que de développer des interactions. Ainsi, les étudiants interviennent longuement ${ }^{6}$ mais ne produisent qu'un seul message. Cela ne les empêche pas parfois de faire écho aux messages précédents ("comme l'a dit Florence, je partage l'avis de Lydie"). On note dans ces écrits un faible dialogue avec les sources et peu de conceptualisation. Les analyses du premier forum restent générales. À l'inverse, les analyses du second forum apparaissent plus approfondies. Les étudiants transfèrent, avec plus ou moins d'habileté et de liberté, les contenus et la méthodologie d'analyse proposés par l'enseignante. Cet apport est manifeste concernant la clarté des notions utilisées et la progression dans les analyses.

\subsubsection{Des cours aux forums : quelle prise en compte des apports des cours dans les messages?}

Du cours A au forum 1

Les écrits du forum 1 se partagent en deux catégories :

- d'une part, ceux minoritaires (4 sur 12) qui rendent compte d'une véritable lecture et d'une vraie appropriation du cours;

- d'autre part, ceux majoritaires (8 sur 12) dont l'analyse propose des apports minimaux qui s'appuient sur quelques éléments succincts du cours $A$, sans référence théorique. Les considérations restent souvent générales et peu pertinentes : "il permet de découvrir la vie historique de Napoléon plutôt comme une bibliographie interactive, à construire et à découvrir par les élèves" (Virginie).

L'absence de consigne explicite sur la nécessité d'utiliser un cours précis, la distance temporelle entre les cours (mis en ligne en novembre) et le forum (ouvert en janvier) jouent sans doute dans cette absence de prise en compte. Pour les formateurs, les cours, les forums et l'écrit de recherche constituent un continuum : les cours sont conçus comme sources de savoirs et de questionnements, à s'approprier, à interroger et à développer dans les forums, de manière à élaborer une réflexion à poursuivre et à approfondir dans l'écrit de recherche. Or ce continuum, implicite, n'est pas compris par les étudiants, pas plus que le rôle du forum dans ce continuum. L'articulation imaginée par le responsable du parcours entre le contenu des cours, les activités du forum et l'écrit de recherche n'est perçue que par une minorité d'étudiants.

Du cours B au forum 2

Contrairement au cas précédent, le cours B a été systématiquement utilisé comme appui pour l'analyse du support. Trois raisons peuvent l'expliquer : la consigne invitant, sinon prescrivant très explicitement de s'appuyer sur celui-ci pour faire l'analyse demandée; la proximité temporelle entre le cours et le forum ouvert le même jour ainsi que l'activité attendue. Il s'agissait en effet de reprendre la méthodologie d'analyse

6310 mots en moyenne pour la première analyse (forum 1) et 582 mots pour la seconde (forum 2). 
appliquée à un manuel pour la transférer sur un support d'apprentissage numérique. Les critères étant immédiatement transférables, l'analyse a été produite par l'ensemble des étudiants, souvent en reprenant l'organisation même du cours. Si quelques étudiants s'appuient sur le cours sans bien le comprendre et produisent erreurs et contresens, une majorité exploite les contenus à bon escient. Cependant aucun de ces écrits, et quel que soit le profil de l'étudiant, ne s'appuie sur les travaux pourtant longuement exposés dans le cadre théorique. Tout se passe comme si l'analyse proposée, immédiatement utilisable, était première, l'aspect théorique étant tenu pour inutile.

\section{Du forum à l'écrit de recherche}

Deux catégories d'étudiants sont à nouveau identifiables : les étudiants, majoritaires, qui intègrent peu les échanges du forum et la synthèse de l'enseignante, et ceux qui s'en saisissent et les incorporent à leur réflexion. Chez les étudiants de ce profil, déjà entrés dans la secondarisation, au sens où ils se montrent capables d'élaborer de nouvelles significations à partir des cours, des lectures et des échanges, et maitrisent déjà les critères du genre attendu, le forum permet d'écrire en exploitant et en renforçant des compétences déjà construites. L'apport de l'enseignant sur le forum a bien un statut de savoir légitime, et il est exploité en tant que tel.

\subsubsection{Le forum comme écrit intermédiaire}

Tous les étudiants reprennent plus ou moins dans leur note de recherche ce qui été produit sur les deux forums. De manière générale, ceux qui ne référencent pas leur réponse sur les forums ne le font pas davantage dans l'écrit de recherche. À l'inverse, l'écrit de recherche peut développer, restructurer et approfondir les écrits des forums qui auront alors permis de concevoir et d'élaborer une réflexion, et qui peuvent donc être envisagés comme des écrits intermédiaires.

Ainsi, Amandine propose une première analyse (forum 1) qui prête des effets magiques au support numérique :

"L'élève va pouvoir naviguer d'un document à un autre, d'une période à une autre comme il le souhaite. Il crée son apprentissage". Ces doxas apparaissent encore dans sa seconde analyse (forum 2) : "Il s'agit d'un document qui place l'élève en situation d'acteur de ses savoirs". Mais elle s'appuie à la fois sur le cours et sur les messages de ses pairs: "Je vois que tout le monde a bien travaillé sur le sujet et je vais vous rajouter ma contribution", pour proposer une analyse plus technique : "Les phrases descriptives de l'œuvre sont essentiellement des phrases simples et il y a quelques phrases complexes par juxtaposition ou coordination, etc. Ces textes sont réduits à deux, voire à trois phrases. Il y a très peu de connecteurs... ". Puis, l'analyse s'enrichit dans la note de recherche où les caractéristiques lexicales et morphosyntaxiques du support sont décrites, ce qui lui permet d'anticiper d'éventuels obstacles à la compréhension : "Les données sont implicites. Les informations pertinentes sont noyées dans la masse des autres informations. Les élèves vont devoir lire entre les lignes, faire le tri et garder l'essentiel..." 
Les écrits intermédiaires sont caractérisés par Chabanne et Bucheton (2002, p. 20) par leur "caractère médiat" et par leur "caractère transitoire et lié à des situations précises de travail». Pour Doquet (2011, p.3), contrairement au brouillon, ils n'ont pas nécessairement vocation "à la préparation d'un écrit définitif", mais "sont surtout le témoignage d'une pensée en train de se construire et le lieu du tâtonnement et de la réflexivité qui accompagnent cette construction". II semble donc que les écrits produits sur ces forums relèvent de l'écrit intermédiaire, dans la mesure où ils permettent à la réflexion de se construire. Dans le processus de réflexion comme dans le processus rédactionnel, ce type de forums semble donc jouer un rôle utile pour les étudiants qui ont saisi les enjeux de ce type d'activité.

Cependant, aux conditions dans lesquelles ces forums sont ici mis en œuvre, il est difficile de les considérer comme propédeutiques à l'écrit de recherche, intrinsèquement liés aux appuis théoriques et à leurs usages, se caractérisant pour Rinck, dans la continuité de Reuter et de Lafont-Terranova et Niewese par : "l'objectivation, la nécessité d'établir dans le discours une séparation entre sujet individuel et sujet épistémique, l'intégration des discours autres dans son propre discours, l'argumentation, etc." (Rinck, 2011 p. 6). Pour ceux qui n'ont pas d'emblée compris ces critères, les forums rendent compte de l'évolution d'une réflexion, plus nourrie et rigoureuse, mais inachevée au regard de ces caractéristiques.

\subsubsection{Médiation et coconstruction des inégalités}

Dans cette étude de cas, on note des effets liés à la médiation épistémique. L'apport du cours et la nécessité explicite de s'y référer a favorisé la capacité des étudiants à conceptualiser et à clarifier les notions utilisées. Le discours des étudiants s'éloigne progressivement de la doxa pour se spécialiser. Enrichis par les contenus du cours et par certains échanges, les messages du second forum utilisent un vocabulaire plus spécifique, s'appuient sur des notions plus conceptualisées, ils gagnent en clarté et en cohérence par rapport au premier forum. Les effets se répercutent également sur la production finale des étudiants puisque sept étudiants utilisent les notions du cours dans leur note de recherche finale, quatre le font de manière partielle et une seule étudiante n'y fait aucune référence.

Cependant, l'analyse des messages du second forum montre aussi les effets relatifs de cette médiation notamment sur la capacité des étudiants à s'approprier les références théoriques pour étayer leurs analyses. Alors que la méthodologie d'analyse, proposée par l'enseignante, a été reprise par la plupart des étudiants (3 étudiants reprennent les éléments essentiels du cours dans leurs analyses, 8 le font partiellement, 1 suit pas à pas la méthodologie d'analyse proposée), la partie théorique, en particulier le concept central de "composite" ", n'est jamais reprise, même par

7 Pour une définition de ce concept, voir : Bautier, É., Crinon, J., Delarue-Breton, C. et Marin, B. (2012). Les textes composites : des exigences de travail peu enseignées? Repères, 45, 63-79. 
les étudiants les plus performants. Le forum 2 montre ainsi l'intérêt de proposer de manière explicite des outils et une méthodologie d'analyse, mais il confirme la difficulté des étudiants à appréhender un cadre théorique : le comprendre, en saisir la nécessité, se l'approprier et s'y appuyer pour construire un cadre d'analyse.

Peraya (1999) rappelle que la médiation relative aux contenus doit être envisagée en complémentarité d'une composante relationnelle, il souligne l'importance de la relation pédagogique et du tutorat dans tout dispositif de formation à distance. Comment le formateur est-il intervenu dans ces forums et quels ont été les effets de cette médiation?

Le formateur est peu présent et il n'explicite pas les enjeux du forum, espace où les étudiants peuvent s'entraîner à écrire et à tester leurs analyses qu'ils pourront ensuite reprendre et développer dans leur écrit de recherche. Le formateur, une fois le cours mis en ligne et la consigne lancée, n'est plus intervenu dans le forum sauf pour la synthèse finale. Il conclut par des félicitations sur la qualité des analyses et des encouragements pour la suite du travail puis propose une double synthèse sur les spécificités relatives au support et sur les difficultés potentielles rencontrées par les élèves face à ce type de support.

Les enjeux de l'activité sont de ce fait mal compris par certains étudiants qui ne perçoivent pas l'intérêt des échanges : "Malgré le retard, j'ai rédigé cette analyse sans consulter celles de mes collègues" (Marie) et qui considèrent l'activité comme un exercice scolaire : "Bonjour Madame, je ne sais pas si ma réponse correspond vraiment à vos attentes..." (Clotilde). Ils ne perçoivent pas non plus l'importance de la synthèse proposée par l'enseignante puisque très peu d'étudiants la reprennent dans leur note de recherche. Ce constat pose la question du statut du savoir énoncé dans le forum. Les étudiants ont visiblement accordé plus de valeur au cours qu'au forum qui traitait pourtant directement de l'objet étudié.

La médiation de l'enseignant peut même produire des effets contreproductifs. Dans le second forum, deux étudiants ont produit des contresens. Ainsi, Virginie considère que des organisateurs textuels permettent "aux élèves de faire une progression chronologique, que la présence d'anaphores marque une certaine cohérence dans le texte, et que de ce fait, les textes du support sont "lisibles, clairs, aérés et faciles à lire pour les élèves"", ce qui revient à dire exactement le contraire de la synthèse finale de l'enseignante : " malgré les apparences, ce support est difficile d'accès pour les élèves. Ce support manque de lien, de cohésion. [...] Ce document se présente sous forme d'une accumulation de suite de phrases, [et] l'élève ne peut pas s'appuyer sur une organisation en paragraphes pour construire la chronologie des événements".

En fait, l'enseignante n'informe pas l'étudiante de ses mauvaises interprétations et le fait de féliciter l'ensemble du groupe sur la qualité des productions pouvait laisser croire à l'étudiante que ses analyses, comme celles de ses camarades, étaient pertinentes. Virginie reprend alors les mêmes conclusions erronées dans sa note de recherche. Ainsi, dans ce cas précis, loin d'être efficace, la médiation semble avoir conforté l'erreur. On observe ainsi que le phénomène de la coconstruction de la diffi- 
cultée $^{8}$, pour ces étudiants fragiles dans leurs acquis, peut également jouer dans le cas des forums où le formateur est présent.

Cette étude permet toutefois de percevoir une fonction essentielle du forum : celle d'écrit intermédiaire pour les étudiants qui publient leur analyse, étape capitale du processus de la réflexion comme de l'écriture. En effet, quel que soit le profil des étudiants, on observe que l'écrit de recherche reprend ce qui a été produit sur les deux forums, en faisant une synthèse plus ou moins élaborée et retravaillée selon les cas. L'étude montre que l'apport épistémique constitué par les cours permet d'enrichir la réflexion des étudiants. Cependant, celle-ci reste inaboutie car il semble que l'implicite sur lequel repose la démarche demandée (nourrir successivement ses écrits des apports des cours et des forums tout en s'appuyant systématiquement sur un cadre théorique référencé) ne soit compris que par une minorité d'étudiants. La majorité en retient les aspects les plus concrets ou immédiatement transposables, et en oublie le soubassement théorique. L'intervention de l'enseignante sur ces forums a un effet variable selon les profils d'étudiants : ou bien l'étudiant lui accorde un statut légitime et le prend en compte au même titre qu'un cours, c'est le cas des étudiants les plus autodirigés; ou bien la synthèse proposée par l'enseignante est ignorée, qu'elle soit lue ou pas, le forum apparaissant alors comme un exercice "scolaire" sur lequel il s'agit de "faire son travail" d'étudiant. Dans ce cas, la médiation de l'enseignant peut contribuer, si elle n'est pas suffisamment ajustée, à empêcher l'accès à la secondarisation.

\section{Conclusion}

L'objectif de cette recherche est de mesurer les effets des échanges dans les forums et de la médiation de l'enseignant sur l'écriture de recherche d'un mémoire de master MEEF. Nous avions montré précédemment que lorsque l'enseignant est absent des échanges dans un forum, deux profils d'étudiants se dégagent. D'un côté, une minorité d'étudiants entrent dans le processus de secondarisation et s'emparent des ressources proposées (cours, références théoriques, échanges avec leurs pairs) pour analyser les situations étudiées. De l'autre, une majorité n'arrive pas à s'approprier les sources disponibles pour conceptualiser. Pour le premier type d'étudiants, les forums apparaissent profitables car ils leur permettent de tester leurs analyses et de s'entraîner à l'écriture de recherche. À l'inverse, pour les étudiants qui entretiennent un rapport "premier" au savoir et au langage, le forum ne fait que refléter des difficultés, que l'absence de médiation et de formalisation des savoirs de la part de l'enseignant ne permet pas de surmonter. Cela confirme le potentiel du forum pour favoriser les apprentissages (Bruillard, 2010) notamment parce qu'il offre un espace d'écrits intermédiaires partagé mais montre également que les apports sont différenciés selon les profils d'étudiants

8 Bonnéry (2009) montre (dans un autre contexte) "comment un dispositif pédagogique qui ne cadre pas l'activité de l'élève sur le cheminement intellectuel attendu pour s'approprier le savoir" fabrique de manière passive des inégalités (Scénarisation des dispositifs pédagogiques et inégalités d'apprentissage. Revue française de pédagogie, $167,13-23)$. 
et atteste qu'un contrôle pédagogique faible, comme c'est souvent le cas pour les forums, s'avère défavorable aux étudiants peu autodirigés (Jézégou, 2008). Nous avons alors cherché à comprendre les effets d'une présence plus affirmée de l'enseignant.

L'analyse des forums utilisés dans notre étude de cas a permis d'observer que l'apport de contenus peut enrichir les points de vue et les productions des étudiants mais elle invite aussi à s'interroger sur le statut du forum dans la formation et la représentation que se font les différents acteurs de ce dispositif pédagogique. Les étudiants, dans la situation étudiée, se sont presque exclusivement appuyés sur les cours pour réaliser leurs analyses et leur écrit de recherche et n'ont pas exploité la synthèse proposée par l'enseignante qui traitait pourtant directement du sujet étudié. Pour certains étudiants, en particulier les moins autodirigés, le forum est considéré comme un exercice et non comme un lieu où il est possible de réfléchir, de travailler son écriture et d'interagir. La même interrogation se pose pour l'enseignant. On observe qu'il n'interagit pas véritablement. Il propose des contenus (sous la forme d'un cours) et une synthèse des échanges mais il ne corrige pas les erreurs d'analyse et ne répond pas aux questions des étudiants. Cette absence de médiation relationnelle limite l'engagement et la participation des étudiants (Quintin, 2008) et explique sans doute que la participation des étudiants se réduit à un seul message.

Ces résultats sont à relativiser compte tenu de la faiblesse de l'échantillon mais ils montrent l'intérêt et les limites de l'apport (seul) de contenus, ils démontrent également que la médiation sur la dimension réflexive, c'est-à-dire sur le sujet même dans son rapport à la recherche, à l'écriture et à l'utilisation des forums, apparaissent indispensables pour surmonter les nombreuses tensions à l'œuvre dans l'écriture de recherche (Reuter, 2004) et pour éviter le mécanisme de coconstruction des inégalités universitaires (Bautier et Rayou, 2009).

Georges Ferone georges.ferone@u-pec.fr

Patricia Richard-Principalli patricia.richard-principalli@u-pec.fr

\section{Bibliographie}

Barré-De Miniac, C., Brissaud, C. et Rispail, M. (dir.) (2004). La littéracie : conceptions théoriques et pratiques d'enseignement de la lecture-écriture. Paris : L'Harmattan.

Bautier, É. et Goigoux, R. (2004). Difficultés d'apprentissage, processus de secondarisation et pratiques enseignantes : une hypothèse relationnelle. Revue française de pédagogie, 148, 89-100.

Bautier, É. et Rayou, P. (2009). Les inégalités d'apprentissage. Programmes, pratiques et malentendus scolaires. Paris: PUF. 
Bruillard, É. (dir.) (2010a). Rapport final de l'ERTé CALICO, Communautés d'apprentissage en ligne, instrumentation, collaboration, 160 p. En ligne : <http://www.stef. ens-cachan.fr/version-francaise/recherche/projets/calico-2006-2009-bilan-239142. kjsp>, consulté en avril 2017.

Chabanne, J.-C. et Bucheton, D. (dir.) (2002). Parler et écrire pour penser, apprendre et se construire. L'écrit et l'oral réflexifs. Paris : PUF

Cosnefroy, L. (2010). L'apprentissage autorégulé : perspectives en formation d'adultes, note de synthèse. Savoirs, 23, 9-50.

Coulon, A. et Paivandi, S. (2008). État des savoirs sur les relations entre les étudiants, les enseignants et les IATOSS dans les établissements d'enseignement supérieur. Rapport pour l'OVE. En ligne < http://www.ove-national.education.fr/medias/files/publications/Rapport_OVE_-_Coulon-Paivandi.pdf $>$, consulté en avril 2017.

Crinon, J. et Guigue, M. (2006). Écriture et professionnalisation. Revue française de pédagogie, 156, 117-169.

Delarue-Breton, C. et Crinon, J. (2015). De l'usage des concepts dans les mémoires de master des futurs enseignants. Le français aujourd'hui, 188, 79-88.

Depover, C., De Lièvre, B., Peraya, D., Quintin, J.-J. et Jaillet, A. (dir.) (2011). Le tutorat en formation à distance. Bruxelles, Belgique : De Boeck.

Delarue-Breton, C. (2014). Le mémoire de master MEEF : un nouveau genre universitaire? Diversité, 177, 50-55.

Delarue-Breton, C. et Crinon, J. (2014). Écrire un mémoire en formation d'enseignants : dialogisme et construction de l'identité professionnelle. Writing Research Across Borders III, Feb 2014, Nanterre, France. 2014. En ligne : <https://halshs.archives-ouvertes.fr/halshs-01359704/>, consulté en avril 2017.

Doquet, C. (2011). Les écrits intermédiaires au cycle 3 pour penser et apprendre. Le français aujourd'hui, 174, 57-68.

Ferone, G. (2011). Interagir à distance en formation des maîtres. Recherche et formation, 68, 79-94.

Ferone, G., Richard-Principalli, P. et Crinon, J. (2016). Les supports numériques pour enseigner, quels obstacles? Littératie numérique scolaire et pratiques enseignantes. In Morin, M.-F., Alamargot, D. et Gonçalves, C., Perspectives actuelles sur l'apprentissage de la lecture et de l'écriture. Contributions about learning to read and write. Sherbrooke, Canada : Éditions de l'université de Sherbrooke.

Jézégou A. (2008). Apprentissage autodirigé et formation à distance. Distances et savoirs, 8, 343-364.

Lafont-Terranova, J. et Niwese, M. (2012). Acculturation à l'écriture de recherche et formation à la didactique de l'écriture. Pratiques, 153-154, 115-128.

Mangenot, F. (2002). Forums et formation à distance : une étude de cas. Éducation permanente, 152, 109-119. 
Peraya, D. (1999). Médiation et médiatisation : le campus virtuel. Hermès, 25, 153-167.

Quintin, J.-J. (2008). Accompagnement d'une formation asynchrone en groupe restreint : modalités d'intervention et modèles de tutorat. Sticef 15. Lyon : INRP.

Rabardel, P. et Samurçay, R. (2006). De l'apprentissage par les artefacts à l'apprentissage médiatisé par les instruments. Éducation et formation, 31-60.

Reuter, Y. (2004). Analyser les problèmes de l'écriture de recherche en formation. Pratiques, 121-122, 9-27.

Reuter, Y. (2012). Les didactiques et la question des littéracies universitaires. Pratiques, 153-154, 161-176.

Rinck, F. (2011). Former à (et par) l'écrit de recherche. Quels enjeux, quelles exigences? Le français aujourd'hui, 174, 79-89.

Rochex, J.-Y. et Crinon, J. (2011). La construction des inégalités scolaires. Rennes : PUR.

\section{Abstract}

\section{Discussion forums: mediation and research writing}

ABSTRACT: Writing research papers is a challenging task for students. Sometimes, teachers use distance tutoring and forums to provide support for them. This case study highlights students' views of different types of input in forums and shows the relative effects of teacher mediation which focuses on content only. It stresses the importance of reflective mediation in the tutoring process as a means of overcoming the many pressures involved in the writing of research.

KEYWORDS: distance learning, forum, writing, teacher education 